\title{
A Self-Consistent Vacuum for Misner Space and the Chronology Protection Conjecture
}

\author{
Li-Xin Li and J. Richard Gott, III \\ Department of Astrophysical Sciences, Princeton University, Princeton, NJ 08544
}

(Received September 5, 1997)

\begin{abstract}
In this paper we find a self-consistent vacuum for Misner space. For this vacuum the renormalized stress-energy tensor is zero throughout the Misner space. A point-like particle detector traveling on a timelike geodesic in a Misner space with this vacuum detects nothing. Misner space with this vacuum thus creates no problems for time travel in and of itself but a time traveler may pose a danger to himself and to the spacetime.
\end{abstract}

PACS number(s): 04.62. +v

Recently, many people have considered the problem of time travel [1] 3]. Time travel, i.e. traveling back in time, requires the existence of closed timelike curves (CTCs). In classical general relativity there are many spacetimes with CTCs solving the Einstein equation (for a review see Ref. 晒). But, in quantum field theory in curved spacetime, several calculations reveal that the renormalized stress-energy tensor of the vacuum polarization in a spacetime with CTCs diverges at the Cauchy horizon which separates the region with CTCs from that without closed causal curves, or at the polarized hypersurfaces which are nested inside the Cauchy horizon [5 10]. Misner space is a simple example of spacetime with CTCs, which is obtained from Minkowski space by identifying points that are taken to each other by the Lorentzian boost transformation [11]. Hiscock and Konkowski have calculated the vacuum polarization of a conformally coupled scalar field in Misner space and found that the renormalized stress-energy tensor diverges at the Cauchy horizon [5]. Based on this result, Hawking has postulated the chronology protection conjecture which states that physical laws do not allow the appearance of CTCs [12]. (For counter-examples to this conjecture see [13 [15].) The vacuum state in Hiscock and Konkowski's calculation is the usual Minkowski vacuum with multiple images in the covering space - Minkowski space. This means that this "adapted" Minkowski vacuum (i.e. the Minkowski vacuum with multiple images) is not a self-consistent vacuum state for Misner space because its geometry is locally flat and so the Einstein equation demands that the renormalized stress-energy tensor is zero. In this paper we find a self-consistent vacuum state for Misner space. For such a vacuum the renormalized stress-energy tensor is zero throughout the Misner space. We also discuss the behavior of a point-like particle detector in Misner space with such a vacuum and find no divergent effects on the detector.

Consider Minkowski space $\left(R^{4}, \eta_{a b}\right)$ with the metric $\eta_{a b}$ given by (throughout the paper we use the units $c=G=$ $\hbar=1)$

$$
d s^{2}=-d t^{2}+d x^{2}+d y^{2}+d z^{2},
$$

where $(t, x, y, z)$ are the usual Cartesian coordinates. Making the transformation

$$
t=\xi \sinh \eta, \quad x=\xi \cosh \eta,
$$

the metric is transformed to the Rindler metric

$$
d s^{2}=-\xi^{2} d \eta^{2}+d \xi^{2}+d y^{2}+d z^{2} .
$$

The Rindler coordinates $(\eta, \xi, y, z)$ only cover the right quadrant (R) of the Minkowski space (i.e. the region $x>|t|)$. By reflection $(t, x, y, z) \rightarrow(-t,-x, y, z)$ (or $(\eta, \xi, y, z) \rightarrow(\eta,-\xi, y, z))$, the Rindler coordinates and the Rindler metric can be extended to the left quadrant $(\mathrm{L})(x<-|t|)$. By the reflection with respect to the hypersurfaces $x= \pm t$, or

$$
\eta \rightarrow \tilde{\xi}-i \frac{\pi}{2}, \quad \xi \rightarrow \pm i \tilde{\eta}, \quad y \rightarrow y, \quad z \rightarrow z
$$

the Rindler coordinates can be extended to the future quadrant (F) $(t>|x|)$ and the past quadrant (P) $(t<-|x|)$. The Rindler metric is extended to $\mathrm{F}$ and $\mathrm{P}$ as

$$
d s^{2}=-d \tilde{\eta}^{2}+\tilde{\eta}^{2} d \tilde{\xi}^{2}+d y^{2}+d z^{2} .
$$

Misner space is obtained by identifying $(t, x, y, z)$ with $(t \cosh n b+x \sinh n b, x \cosh n b+t \sinh n b, y, z)$ where $n$ is any integer and $b$ is a positive boost constant. Under such an identification the point $(\eta, \xi, y, z)$ in $\mathrm{R}$ (or $\mathrm{L}$ ) is identified 
with the points $(\eta+n b, \xi, y, z)$ in $\mathrm{R}$ (or L), the point $(\tilde{\eta}, \tilde{\xi}, y, z)$ in $\mathrm{F}$ (or P) is identified with points $(\tilde{\eta}, \tilde{\xi}+n b, y, z)$ in $\mathrm{F}$ (or P). Clearly there are CTCs in R and L but there are no CTCs in F and P, and these regions are separated by the Cauchy horizons $x= \pm t$ which are generated by closed null geodesics.

Usually there is no well-defined quantum field theory in spacetimes with CTCs. However, in the simple case of Misner space, we can do it in the covering space - Minkowski space. In Minkowski space there are two familiar vacuum states: the Minkowski vacuum, which is invariant under the Poincaré group; and the Rindler vacuum, which is not Poincaré invariant but is invariant under the Lorentz boost transformation 16. It is well known that the Minkowski vacuum is a thermal state relative to the Rindler vacuum, a particle detector with constant acceleration moving in the Minkowski vacuum behaves as if it is in a thermal bath [17. Hiscock and Konkowski have calculated the vacuum polarization of a conformally coupled scalar field in the "adapted" Minkowski vacuum for Misner space and found that the renormalized stress-energy tensor diverges at the Cauchy horizon, which has caused Hawking to conclude that such a spacetime cannot be stable against vacuum fluctuations and that therefore the chronology protection conjecture holds. But, this means that the "adapted" Minkowski vacuum is not a self-consistent quantum state for the Misner space because the Einstein equation is not satisfied.

In the case of an eternal Schwarzschild black hole, there are the Boulware vacuum 18 and the Hartle-Hawking vacuum [19]. The globally defined Hartle-Hawking vacuum bears essentially the same relationship to the Boulware vacuum as the Minkowski vacuum does to the Rindler vacuum [20]. For the Boulware vacuum, the renormalized stress-energy tensor diverges at the horizon, which means that this state is not a self-consistent vacuum for the Schwarzschild black hole because when one inserts this stress-energy tensor back into the Einstein equation the back-reaction will completely alter the Schwarzschild geometry near the horizon. For the Hartle-Hawking vacuum however the renormalized stress-energy tensor is finite everywhere and an static observer outside the horizon sees Hawking radiation [21. People usually regard the Hartle-Hawking vacuum as the reasonable vacuum state for an eternal Schwarzschild black hole because when the stress-energy tensor is inserted back into the Einstein equation the Schwarzschild geometry is only altered slightly [22]. Therefore in the case of Misner space we should try to find a vacuum which is self-consistent. Let us consider the Rindler vacuum. The Hadamard function for a conformally coupling scalar field in the Rindler vacuum is [23]

$$
G_{R}^{(1)}\left(X, X^{\prime}\right) \equiv\left\langle 0_{R}\left|\phi(X) \phi\left(X^{\prime}\right)+\phi\left(X^{\prime}\right) \phi(X)\right| 0_{R}\right\rangle=\frac{1}{2 \pi^{2}} \frac{\gamma}{\xi \xi^{\prime} \sinh \gamma\left[-\left(\eta-\eta^{\prime}\right)^{2}+\gamma^{2}\right]},
$$

where $X=(\eta, \xi, y, z), X^{\prime}=\left(\eta^{\prime}, \xi^{\prime}, y^{\prime}, z^{\prime}\right)$ and $\gamma$ is defined by

$$
\cosh \gamma=\frac{\xi^{2}+\xi^{2}+\left(y-y^{\prime}\right)^{2}+\left(z-z^{\prime}\right)^{2}}{2 \xi \xi^{\prime}}
$$

$G_{R}^{(1)}$ in Eq.(6) is well-defined in region R and can be analytically extended to region L by $(\eta, \xi, y, z) \rightarrow(\eta,-\xi, y, z)$, and regions $\mathrm{F}$ and $\mathrm{P}$ by the transformations in (4). Using the method of images, the corresponding Hadamard function in Misner space is

$$
G^{(1)}\left(X, X^{\prime}\right)=\sum_{n=-\infty}^{\infty} \frac{1}{2 \pi^{2}} \frac{\gamma}{\xi \xi^{\prime} \sinh \gamma\left[-\left(\eta-\eta^{\prime}+n b\right)^{2}+\gamma^{2}\right]} .
$$

The Hadamard function for the usual Minkowski vacuum is

$$
G_{M}^{(1)}\left(X, X^{\prime}\right)=\frac{1}{2 \pi^{2}} \frac{1}{-\left(t-t^{\prime}\right)^{2}+\left(x-x^{\prime}\right)^{2}+\left(y-y^{\prime}\right)^{2}+\left(z-z^{\prime}\right)^{2}} .
$$

As usual the regularized Hadamard function is taken to be

$$
G_{\text {reg }}^{(1)}=G^{(1)}-G_{M}^{(1)}
$$

The renormalized stress-energy tensor is obtained from the Hadamard function by [24,25]

$$
\left\langle T_{a b}\right\rangle_{\mathrm{ren}}=\frac{1}{2} \lim _{X^{\prime} \rightarrow X}\left(\frac{2}{3} \nabla_{a} \nabla_{b^{\prime}}-\frac{1}{3} \nabla_{a} \nabla_{b}-\frac{1}{6} g_{a b} \nabla_{c} \nabla^{c^{\prime}}\right) G_{\mathrm{reg}}^{(1)} .
$$

Inserting Eqs.(8-10) into Eq.(11), we obtain the renormalized stress-energy tensor for the vacuum with the Hadamard function in (8) 


$$
\left\langle T_{a b}\right\rangle_{\mathrm{ren}}=\frac{1}{1440 \pi^{2} \xi^{4}}\left[\left(\frac{2 \pi}{b}\right)^{4}-1\right]\left(4 \xi^{2} d \eta_{a} d \eta_{b}+g_{a b}\right)
$$

This is the renormalized stress-energy tensor in R. We find that unless $b=2 \pi,\left\langle T_{a b}\right\rangle_{\text {ren }}$ blows up as one approaches the Cauchy horizon $(\xi \rightarrow 0)$. But, for the case $b=2 \pi$ we have

$$
\left\langle T_{a b}\right\rangle_{\mathrm{ren}}=0,
$$

which is regular on the Cauchy horizon and can be regularly extended to regions $\mathrm{L}, \mathrm{F}$, and $\mathrm{P}$ where it is also zero. With such a vacuum state (whose Hadamard function is given by Eq.(8) with $b=2 \pi$ ), the Misner space is self-consistent i.e. the vacuum Einstein equation is satisfied exactly. Therefore such a vacuum state is self-consistent and $b=2 \pi$ is the self-consistent condition. (Another solution for $\left\langle T_{a b}\right\rangle_{\mathrm{ren}}=0$ in Eq.(12) is $b=i 2 \pi$ which is just the usual Minkowski vacuum expressed as a thermal state relative to a Rindler observer. Periodicity in imaginary time is a characteristic of a thermal Green function [26,27].)

Another way to deal with quantum fields in spacetimes with CTCs is to do the quantum field theory in the Euclidean section and then analytically extend the results to the Lorentzian section [28]. For Misner space the Euclidean section is obtained by taking $\eta$ and $b$ to be $-i \bar{\eta}$ and $-i \bar{b}$. The resultant space is the Euclidean space with metric $d s^{2}=\xi^{2} d \bar{\eta}^{2}+d \xi^{2}+d y^{2}+d z^{2}$ and $(\bar{\eta}, \xi, y, z)$ and $(\bar{\eta}+n \bar{b}, \xi, y, z)$ are identified where $(\bar{\eta}, \xi, y, z)$ are cylindrical polar coordinates with $\bar{\eta}$ the angular polar coordinate and $\xi$ the radial polar coordinate. The geometry at the hypersurface $\xi=0$ is conical singular unless $\bar{b}=2 \pi$. When extending that case to the Lorentzian section, we get $b=2 \pi$ which is just the self-consistent condition. This may be the geometrical explanation of the self-consistent condition. By doing quantum field theory in the Euclidean space, then analytically extending the results to the Lorentzian section, we obtain the renormalized stress-energy tensor in R (or L) region of the Misner space. Then we can extend the renormalized stress-energy tensor in $\mathrm{R}$ (or L) to regions $\mathrm{F}$ (or P). The results are the same as that obtained with the method of images.

Now we have a self-consistent vacuum state for the conformally coupled scalar field in the Misner space satisfying the self-consistent condition, whose Hadamard function is given by Eq.(8) with $b=2 \pi$. If the quantum field is in such a state, the renormalized stress-energy tensor vanishes everywhere, and the Einstein equation is satisfied exactly. In order to see if Misner space in such a quantum state allows time travel, we consider a point-like particle detector moving in this space. The detector is assumed to be in its ground state originally. The probability that the detector is excited is given by the response function [25]

$$
\mathcal{F}(E)=\int_{-\infty}^{\infty} d \tau \int_{-\infty}^{\infty} d \tau^{\prime} e^{-i E\left(\tau-\tau^{\prime}\right)} G^{+}\left(X(\tau), X\left(\tau^{\prime}\right)\right)
$$

where $\tau$ is the proper time of the detector, $X(\tau)$ is the worldline of the detector, $E(>0)$ is the difference of energy between the excited state and the ground state of the detector, $G^{+}\left(X, X^{\prime}\right) \equiv\left\langle\left|\phi(X) \phi\left(X^{\prime}\right)\right|\right\rangle$ is the Wightman function. Suppose the detector moves along a geodesic with $x=a, y=\beta_{y} t$, and $z=0$ ( $a$ and $\beta_{y}$ are constants and $a$ is positive), which goes through the $\mathrm{P}, \mathrm{R}$, and $\mathrm{F}$ regions. The proper time of the detector is $\tau=t / \zeta$ with $\zeta=1 / \sqrt{1-\beta_{y}^{2}}$. Along the geodesic, the Hadamard function in (8) is reduced to

$$
G^{(1)}\left(t, t^{\prime}\right)=\frac{1}{2 \pi^{2}} \frac{\gamma}{\sinh \gamma \sqrt{\left(a^{2}-t^{2}\right)\left(a^{2}-t^{\prime 2}\right)}} \sum_{n=-\infty}^{\infty} \frac{1}{-\left(\eta-\eta^{\prime}+n b\right)^{2}+\gamma^{2}},
$$

where $\gamma$ is given by

$$
\cosh \gamma=\frac{2 a^{2}-t^{2}-t^{2}+\beta_{y}^{2}\left(t-t^{\prime}\right)^{2}}{2 \sqrt{\left(a^{2}-t^{2}\right)\left(a^{2}-t^{\prime 2}\right)}}
$$

and $\eta-\eta^{\prime}$ is given by

$$
\sinh \left(\eta-\eta^{\prime}\right)=\frac{a\left(t-t^{\prime}\right)}{\sqrt{\left(a^{2}-t^{2}\right)\left(a^{2}-t^{\prime 2}\right)}} .
$$

Though this Hadamard function is originally defined only in $\mathrm{R}$ it can be analytically extended to F, $\mathrm{P}$, and $\mathrm{L}$. The Wightman function is equal to $1 / 2$ of the Hadamard function with $t$ replaced by $t-i \epsilon / 2$ and $t^{\prime}$ replaced by $t^{\prime}+i \epsilon / 2$ where $\epsilon$ is an infinitesimal positive real number. Then the response function is 


$$
\begin{aligned}
& \mathcal{F}(E)=\frac{1}{4 \pi^{2}} \sum_{n=-\infty}^{\infty} \int_{-\infty}^{\infty} d T \int_{-\infty}^{\infty} d \Delta \tau \\
& \frac{\gamma^{+} e^{-i E \Delta \tau}}{\sinh \gamma^{+} \sqrt{\left[a^{2}-\zeta^{2}\left(T+\frac{\Delta \tau}{2}-\frac{i \epsilon}{2 \zeta}\right)^{2}\right]\left[a^{2}-\zeta^{2}\left(T-\frac{\Delta \tau}{2}+\frac{i \epsilon}{2 \zeta}\right)^{2}\right]}\left\{-\left[\left(\eta-\eta^{\prime}\right)^{+}+n b\right]^{2}+\gamma^{+^{2}}\right\}},
\end{aligned}
$$

where $T \equiv\left(\tau+\tau^{\prime}\right) / 2, \Delta \tau \equiv \tau-\tau^{\prime} ; \gamma^{+}$and $\left(\eta-\eta^{\prime}\right)^{+}$are given by (16) and (17) with $t$ replaced by $t-i \epsilon / 2$ and $t^{\prime}$ replaced by $t^{\prime}+i \epsilon / 2$. The integral over $\Delta \tau$ can be worked out by the residue theorem where we choose the integration contour to close in the lower half complex- $\Delta \tau$ plane. The result is zero since there are no poles in the lower half plane. Therefore such a detector cannot be excited and so it detects nothing. It is interesting to note that such a conclusion holds even for a simply connected Minkowski space: An inertial particle detector moving in the Rindler vacuum detects nothing though the Rindler vacuum is different from the Minkowski vacuum. This can be explained by the fact that the energy level of the Rindler vacuum is below that of the Minkowski vacuum [29] therefore the Rindler vacuum cannot excite an inertial detector in its ground state. We have also calculated the response functions for detectors on worldlines with constant $\xi, y$, and $z$ and worldlines with constant $\tilde{\xi}, y$, and $z$, both are zero.

However, in this particular spacetime a time traveler may pose a danger to himself - and to the spacetime as a whole. The time traveler will collide with himself unless his worldline is carefully arranged. A time traveler moving along the geodesic discussed above will hit himself unless $\beta_{y}$ is non-zero and his dimension is sufficiently small compared with $a$. In the covering space an infinite number of images of the time traveler must pass inside the sphere $x^{2}+y^{2}+z^{2}=a^{2}$ at $t=0$. So unless the total mass of the time traveler and his spaceship is exactly zero it will distort the geometry of the spacetime and the exact solution will not apply. This may be avoided if the time traveler and his spaceship are arranged to have a spherically symmetric mass distribution and are surrounded by a negative mass shell so that the total mass is zero and the gravitational field outside the shell is zero. (This would require violating the weak energy condition as is done in any case in the wormhole solution [1]). The time traveler must also not emit any stray photons that he does not later re-absorb.

Misner space suffers from classical instability in the sense that a propagating wave may pile up on the Cauchy horizon and destroy the Cauchy horizon due to the infinite blue-shift of the frequency of the wave [1]. This is similar in nature to the perturbation on Misner space caused by a positive mass time traveler discussed above. However, this classical instability can be avoided in the wormhole spacetime with CTCs due to the divergent effect of the wormhole [1]. (The relation between Misner space and the wormhole spacetime with CTCs can be found in 《]).

Grant space is identical to Misner space except that in Grant space there is a shift in the $y$ direction when one makes the identification in Minkowski space [10]. Grant space is identical to Gott's two-string spacetime far from the strings 10,30 but different in that Gott space has some Casimir effects that Grant space does not. In Grant space the problem of the time traveler hitting himself (e.g. for a time traveler with $x=a$ and $y=z=0$ ) is avoided if the dimension of the time traveler is less than the shift in the $y$ direction. But Thorne has conjectured that black holes with horizons form when and only when a mass $M$ gets compacted into a region whose circumference in every direction is $\mathcal{C}<4 \pi M$ [31. At $t=0$, the pair of $n$-th and $(-n)$-th images of the time traveler form a system with total mass $M=2 m \cosh n b$ in their center of mass frame, where $m$ is the rest mass of the time traveler. The circumference is $\mathcal{C}=4 n y_{0}$ where $y_{0}$ is the shift in the $y$ direction. If $m>0$ one can always find an $n$ large enough so that $4 n y_{0}<4 \pi \cdot 2 m \cosh n b$ and $\mathcal{C}<4 \pi M$ thus causing a black hole to form according to Thorne's conjecture. Then the spacetime would be significantly perturbed perhaps eliminating the CTCs. Therefore Grant space appears to be classically unstable to a perturbation by a positive mass time traveler. And so also by implication is Gott's two-string spacetime with CTCs. For the finite string loop case, Gott's two-string spacetime with CTCs already satisfies Thorne's limit for black hole formation, suggesting that the CTCs would either be destroyed or hidden behind an event horizon [3,32]. We have found a self-consistent vacuum for Grant space only in the case of $y_{0}=0$, but in such a case Grant space is reduced to Misner space.

We have also found a self-consistent vacuum for the multiply connected de Sitter space which is obtained from the usual de Sitter space by identifying points that are taken to each other by the de Sitter-boost transformation, which will be discussed elsewhere [33]. In such a case the renormalized stress-energy tensor is non-zero but the Einstein equation is exactly solved for some specific cosmological constant (as in [34]).

\section{ACKNOWLEDGMENTS}

This research was supported by NSF grant AST95-29120. 
Note added (11/24/97): It has just come to our attention that M. J. Cassidy (Class. Quantum Grav. 14, 3031 (November 1997)) has independently argued, using Euclidean methods and starting from the vacuum around a cosmic string, that a solution with $\left\langle T_{a b}\right\rangle_{\mathrm{ren}}=0$ must exist for a conformal scalar field in Misner space where $b=2 \pi$ (in agreement with our result). He didn't propose what quantum state it would correspond to (we have found here that it is an "adapted" Rindler vacuum), but he noted correctly that it would have to be different than the Hiscock and Konkowski vacuum. We agree with C. R. Cramer and B. S. Kay's (Class. Quantum Grav. 13, L143 (1996)) conclusion (in the automorphic field [15] case) that $\left\langle T_{a b}\right\rangle_{\text {ren }}$ remains formally ill-defined on the Misner space Cauchy horizon itself, but we note that this may not be a problem in physics since this is a set of measure zero, and $\left\langle T_{a b}\right\rangle_{\mathrm{ren}}=0$ everywhere else.

[1] M. S. Morris, K. S. Thorne, and U. Yurtsever, Phys. Rev. Lett. 61, 1446 (1988).

[2] V. P. Frolov and I. D. Novikov, Phys. Rev. D 42, 1057 (1990).

[3] J. R. Gott, Phys. Rev. Lett. 66, 1126 (1991).

[4] K. S. Thorne, in General Relativity and Gravitation 1992: Proceedings of the 13th International Conference on General Relativity and Gravitation, edited by R. J. Gleiser, C. N. Kozameh and O. M. Moreschi (IOP Publishing, Bristol, 1993), p. 295.

[5] W. A. Hiscock and D. A. Konkowski, Phys. Rev. D 26, 1225 (1982).

[6] S. -W. Kim and K. S. Thorne, Phys. Rev. D 43, 3929 (1991).

[7] V. P. Frolov, Phys. Rev. D 43, 3878 (1991).

[8] D. G. Boulware, Phys. Rev. D 46, 4421 (1992).

[9] G. Klinkhammer, Phys. Rev. D 46, 3388 (1992).

[10] J. D. E. Grant, Phys. Rev. D 47, 2388 (1993).

[11] C. W. Misner, in Relativity Theory and Astrophysics I: Relativity and Cosmology, edited by J. Ehlers, Lectures in Applied Mathematics, Vol. 8 (American Mathematical Society, Providence, 1967), p. 160.

[12] S. W. Hawking, Phys. Rev. D 46, 603 (1992).

[13] L. -X. Li, Phys. Rev. D 50, R6037 (1994).

[14] L. -X. Li, Class. Quantum Grav. 13, 2563 (1996).

[15] S. V. Sushkov, Class. Quantum Grav. 14, 523 (1997).

[16] S. A. Fulling, Phys. Rev. D 7, 2850 (1973).

[17] W. G. Unruh, Phys. Rev. D 14, 870 (1976).

[18] D. G. Boulware, Phys. Rev. D 11, 1404 (1975).

[19] J. B. Hartle and S. W. Hawking, Phys. Rev. D 13, 2188 (1976).

[20] D. W. Sciama, P. Candelas, and D. Deutsch, Adv. Phys. 30, 327 (1981).

[21] P. Candelas, Phys. Rev. D 21, 2185 (1980).

[22] J. W. York, Phys. Rev. D 31, 775 (1985).

[23] J. S. Dowker, Phys. Rev. D 18, 1856 (1978).

[24] R. M. Wald, Phys. Rev. D 17, 1477 (1978).

[25] N. D. Birrell and P. C. W. Davies, Quantum Fields in Curved Space (Cambridge University Press, Cambridge, 1982).

[26] A. L. Fetter and J. D. Walecka, Quantum Theory of Many-Particle Systems (McGraw-Hill, San Francisco, 1971).

[27] G. W. Gibbons and M. J. Perry, Proc. R. Soc. Lond. A 358, 467(1978).

[28] S. W. Hawking, Phys. Rev. D 52, 5681 (1995).

[29] P. Candelas and D. Deutsch, Proc. R. Soc. Lond A 354, 79 (1977).

[30] D. Laurence, Phys. Rev. D 50, 4957 (1994).

[31] K. S. Thorne, in Magic without Magic: John Archibald Wheeler, edited by J. R. Klauder (W. H. Freeman and Company, San Francisco, 1972), p. 231.

[32] M. P. Headrick and J. R. Gott, Phys. Rev. D 50, 7244 (1994).

[33] J. R. Gott and L. -X. Li, in preparation.

[34] J. R. Gott, Nature 295, 304 (1982). 